\title{
The Construct Validity and Responsiveness of Sensory Tests in Patients with Carpal Tunnel Syndrome
}

\author{
Derek K.M. Cheung ${ }^{*}, 1$, Joy C. MacDermid ${ }^{2,3}$, Dave Walton ${ }^{1}$ and Ruby Grewal ${ }^{2,4}$ \\ ${ }^{1}$ Health and Rehabilitation Sciences Physical Therapy, Faculty of Health Sciences, Western University, London, \\ Ontario, Canada \\ ${ }^{2}$ Hand and Upper Limb Clinic, St. Joseph's Hospital, London, Ontario, Canada \\ ${ }^{3}$ School of Rehabilitation Science, McMaster University, Hamilton, Ontario, Canada \\ ${ }^{4}$ Schulich School of Medicine, Western University, London, Ontario, Canada
}

\begin{abstract}
Background and Purpose: Sensory evaluation is fundamental to evaluation of patients with Carpal Tunnel Syndrome (CTS). The purpose of this study was to determine the construct validity and responsiveness for sensory threshold tests in patients with CTS.

Methods: Sixty-three patients diagnosed with CTS were evaluated prior to orthotic intervention and again at follow up at 6 and 12 weeks. Sensory tests included touch threshold PSSD (Pressure Specified Sensory Device) and vibration threshold (Vibrometer). Construct validity was assessed by comparing sensory tests to hand function, and dexterity testing using Spearman rho $\left(\mathrm{r}_{\mathrm{s}}\right)$. Patients were classified as either responders or non-responders to orthotic intervention based on the change score of the Symptom Severity Scale (SSS) of 0.5. Responsiveness of the sensory tools was measured using ROC (receiver operating characteristic) curves, SRM (Standardized Response Mean), and ES (Effect Sizes).

Results: The PSSD had low to moderate correlations $\left(\mathrm{r}_{\mathrm{s}} \leq 0.32\right)$ while Vibrometer scores had moderate correlations $\left(\mathrm{r}_{\mathrm{s}}=0.36-0.41\right)$ with dexterity scores. The Clinically Important Difference (CID) for the PSSD was estimated at $0.15 \mathrm{~g} / \mathrm{mm}^{2}$ but was not discriminative. The Vibrometer demonstrated moderate responsiveness, with a SRM $=0.61$ and an $\mathrm{ES}=0.46$ among responders. The PSSD had a $\mathrm{SRM}=0.09$ and an $\mathrm{ES}=0.08$ and showed low responsiveness for patients with a clinically important improvement in symptoms.

Conclusion: Measurement properties suggest that the Vibrometer was preferable to the PSSD because it was more correlated to hand function, and was more responsive. Clinicians may choose use the Vibrometer opposed to the PSSD for determining important change in sensation after orthotic intervention.
\end{abstract}

Keywords: Carpal tunnel syndrome, clinically important difference, construct validity, orthotic intervention, psychometric properties, responsiveness, touch threshold, vibration threshold.

\section{INTRODUCTION}

Carpal Tunnel Syndrome (CTS) is a common compression neuropathy with an annual prevalence ranging between $2.7 \%$ [1] to $6.7 \%$ [2]. Occupations with repetitive motion (for example, manufacturing and food services), have higher prevalence of CTS cases in the United States of America based on National Health Surveys [2, 3]. For workers employed within educational institutions, a lower prevalence of CTS injury was found [2]. CTS is a major component of work-relevant upper extremity disorders $[4,5]$, and is associated with considerable health care costs [6]. Typical sensory symptoms experienced by patients with CTS are tingling, pain, and numbness at night or throughout the day; with advanced stages of the disease affecting motor

*Address correspondence to this author at the Hand and Upper Limb Centre, Clinical Research Laboratory (Basement), St. Joseph's Health Centre, 268 Grosvenor St., London, Ontario, N6A 4L6, Canada; Tel: 519-646-6000; Fax: 519-646-6049; E-mail: derek.kmcheung@gmail.com function and dexterity [7-14]. Sensory findings are early indicators of CTS and commonly evident in the clinical history. In addition, clinical examination tests of sensibility and hand function are used to assess sensibility and its functional impact [15-20]. This includes sensory tests [21], or disease specific questionnaires [22]. These clinical tests should be responsive, such that they demonstrate noticeable change to the patient or to the clinician [23]. Responsiveness is central to the confidence that clinicians and patients have in using clinical tests to determine whether treatment was successful; and if further treatment is required afterwards [15].

There are two broad methods of determining clinically meaningful change: distribution based and anchor based approaches. Distribution based methods focus on statistical properties of a tool for measuring clinically important change [24]. Two common methods of distribution based methods are effect size (ES) and standardized response mean (SRM) [25]. Anchor based methods define clinically 
important change based on an external anchor, which can be based on a subjective opinion (from a clinician's or patient's perception) or from an objective measure (such as a disease specific tool) [25]. The anchor must be clearly defined and be able to show a clinically important difference (CID) between groups at one instant (cross sectional approach) or over a period of time (longitudinal approach) [24]. Often, a global rating of change is used to determine subjective change. Although this method is easy to apply, it is subject to recall bias [26]. The use of a disease specific tool is thought to be a more accurate to measure important change because they are standardized, contain multiple items, and are more prescriptive about what elements are being evaluated. Another criterion for clinically important change is whether symptoms resolve with treatment. The benefit of this approach is that it is an external criterion for demonstrating clinical improvement. For example, if symptoms continue despite conservative treatment, the patient did not experience a clinical important change, and surgery would be the next treatment option [27].

Sensation is an important component of hand function $[19,28]$, and is typically impaired in CTS [29]. A variety of tools can provide quantitative measures of sensory threshold for different modalities, including touch and vibration threshold [15-17, 19]. Two sensory tools which are used in clinical studies to measure sensory characteristics in CTS are the Pressure Specified Sensory Device (PSSD) (NK Biotechnical Corporation, Minneapolis, MN, USA) and the Vibrometer ( $Z$ tech Medical, Salt Lake City, UT, USA). Some clinical measurement properties of the PSSD and Vibrometer have been reported. The PSSD has been shown to have high reliability $(\mathrm{r}=0.95)$ and inter rater reliability in healthy persons for one point static testing $(r=0.99)$ [30]. The Vibrometer, has shown to have excellent test-retest reliability in patients with CTS with intra class correlation coefficients (ICCs) ranging from 0.86 to 0.89 [31]. However, there is a lack of studies defining the responsiveness (CID, ES, and SRM) of the sensory tools. Valid sensory measures should be related to measures of hand function, such as dexterity, and self-reported function as measured by the DASH (Disability of the Arm, Shoulder, and Hand).

The purpose of this study was to evaluate the clinical measurement properties of the Vibrometer and the PSSD in patients with CTS, in terms of the following:

1. The responsiveness using:

a. Distribution based - effect size (ES) and standardized response means (SRM)

b. Anchor based clinically important difference (CID)

2. The convergent construct validity in relation to measures of self-reported and performance-based hand function (DASH and NK Dexterity).

\section{MATERIALS AND METHODOLOGY}

\section{Participants}

All patients were recruited from a tertiary care center specializing in upper limb disorders. Patients were diagnosed with CTS based on a clinical diagnosis made by the treating hand surgeons and confirmed by electromyography (EMG) from various testing centers based on the latest version of the American Association of Electrodiagnostic Medicine criteria as outlined by consensus criteria by Rempel et al. (Table 1a) [14]. Patients were excluded from the study if they met any of the following criteria:

\section{Exclusion Criteria:}

- $\quad$ urgent or severe CTS requiring early operative intervention

- $\quad$ pregnancy [32-34]

- $\quad$ concurrent injury to the upper extremity including recent trauma (i.e. fracture, amputation, tumor, or nerve compression)

- wrist arthritis, rheumatoid arthritis diabetes mellitus, or thyroid disease

- $\quad$ inability to complete study forms/assessments

- neurodegenerative conditions which prevent the participant from fully understanding and completing the research study

This study was approved by the university research ethics board and informed consent was obtained from each patient.

\section{Sample Size Justification}

The sample size was determined for the correlation between sensory tools to DASH, and Dexterity to achieve significance using $\mathrm{G}^{*}$ Power version 3.1.4 software (http: //www.psycho.uni-duesseldorf.de/abteilungen/aap/gpower3/dow nload-and-register) at a power of $80 \%$ with a moderate effect size of 0.5 with a point biserial model. Alpha was set at 0.05 using a 2 tail test. The minimum sample size required was 26 patients. Statistical significance was considered if $p<0.05$.

\section{Outcome Measures}

\section{Pressure Specified Sensory Device (PSSD)}

The PSSD is a computerized touch threshold device, which can measure the minimum amount of pressure required to elicit a response from a subject $\left(\mathrm{g} / \mathrm{mm}^{2}\right)$, as well as spatial discrimination (2 point discrimination) ( 2 PD). It has a range of 0.1 to $100 \mathrm{~g} / \mathrm{mm}^{2}$. Each hemispheric prong has an area of $0.90 \mathrm{~mm}^{2}$. This study tested touch threshold only with the PSSD. For touch threshold of a single point, the tester applied an individual metallic prong from the PSSD device into the distal pulp of the long finger in the affected hand while the participant sat with eyes closed. The system calibrates itself each time the software is started to ensure reliability. Participants were instructed to push a trigger held in the opposite hand, to identify when they perceived the stimulus. The PSSD has been shown to have high reliability $(\mathrm{r}=0.95)$ and inter-test reliability in patients with neuropathy for one point static testing $(r=0.99)$ [30]. For each visit, a total of five repetitions were taken; the lowest and highest scores were dropped and the remaining three were averaged as recommended by the manufacturer. 


\section{Vibrometer}

The Vibrometer is a sensory modality which measures vibration perception threshold [35]. The Vibrometer used in this study is a $50 \mathrm{~Hz}$ computer-controlled ramped protocol where the vibration stimuli are applied through a $2 \mathrm{~mm}$ diameter aperture with a $1 \mathrm{~mm}$ diameter vibrating post (Fig. 1). Subjects were required to identify when they felt a stimulus by squeezing a handheld trigger with their eyes closed. The minimum score is $1 \mathrm{um}$ and the maximum score is $180 \mathrm{um}$. A ramped protocol is regulated by the device supplying sufficient repetitions of test stimuli; to achieve a stable estimate of vibration threshold. The Vibrometer has shown excellent test retest reliability in patients with CTS with ICCs ranging from 0.86 to 0.89 [31].



Fig. (1). Vibrometer apparatus with trigger.

\section{Disability of the Arm, Shoulder, and Hand (DASH) Questionnaire}

The DASH is a self-report measure which allows patients to rate the disability of their arm, shoulder, and hand [32]. The questionnaire contains 30 questions rated 1 to 5 , with a higher score indicating higher levels of pain and disability. The DASH was completed by each patient at each follow up point. The final DASH score was calculated by adding all the scores, subtracts 30 , and divided by 1.20 . The questionnaire has been shown to be responsive after carpal tunnel release surgery $(\mathrm{SRM}=0.7)[33]$. The convergent, construct, and discriminatory validity has been supported for distal upper extremities, including CTS [34]. DASH has also been found to be responsive in patients with wrist related injuries $(\mathrm{SRM}=0.74-0.91)$ [34].

\section{NK Dexterity Small Objects Test}

The NK Dexterity Small Objects Test (referred to as "Dexterity" for short) is a test of manual dexterity which measures the amount of time (in seconds) that is required for a patient to move objects on a plastic board [36, 37]. Sizes of the objects are classified as small, medium, or large. It consists of plastic and metal objects that need to be moved with the affected hand and placed into another location. Timing was initiated from the moment the hand moved from the starting position towards the first object until subjects removed their hand from the final object. For this study, dexterity testing was done with the small objects only because fine motor function is expected to be most affected in CTS [36]. In addition, the small object subtest has been shown to have the best correlation to hand function with $\mathrm{r}=0.47-0.87$; and also has high reliability $(\mathrm{ICC}=0.53-0.86)$ $[36,37]$. The time recorded for the patient to complete the dexterity task was the mean of 3 trials at each follow up point.

\section{Symptom Severity Scale (SSS)}

The Symptom Severity Scale is a validated patient oriented scale to assess the symptoms and functions experienced by patients with CTS. The questionnaire is composed of two parts. Eleven questions are used to measure the symptoms experienced by patients and 8 questions are used to measure function [22]. Each question is rated from 1 to 5. The average of all scores was taken as the final score of the test. The tool has been validated for responsiveness, and reliability [22].

\section{Procedure}

Each patient was treated with night orthotic intervention of the wrist in neutral position for 12 weeks; and had assessments at baseline, 6 weeks, and 12 weeks later (total of 3 visits). All patients had received orthopedic intervention regardless of severity, as this is standard treatment practice in this tertiary care setting. If patients and surgeons felt that orthotic intervention was not reducing symptoms, then surgery was offered following the 12 weeks. Patients were monitored for a year following the intervention to determine whether they proceeded to surgery.

At baseline and at each follow up visit, patients completed the following assessments: PSSD, Vibrometer, DASH, and Dexterity. The same research assistant was trained to perform each of the tests on each patient and at each follow up point. Data for the PSSD and Vibrometer were collected by having each patient seated with their affected arm supported on a table. For the PSSD, the wrist was supported by a piece of foam, such that the wrist was in neutral position and the palm was facing upwards. Data for the Vibrometer was collected by having the arm rest directly on the table with the hand in pronated position. The long finger was used for testing sensation in both PSSD and Vibrometer testing. Testing was done on the affected hand if CTS was affecting only one side; otherwise, the hand with more severe symptoms was tested for subjects who had CTS in both hands. Standardized testing was done for dexterity testing for each patient with the affected hand.

\section{Analysis}

All analyses were performed using SPSS software version 19.

\section{Cross Sectional Convergent Validity}

Cross Sectional Convergent Validity assesses the extent to which a measure's result agrees with another measure that is believed to be assessing the same or similar attribute [38]. 
The correlation between the PSSD and the Vibrometer to measures of hand function were determined using Spearman's correlation $\left(r_{s}\right)$. Interpretation was based on the guidelines that Spearman' correlation are considered poor if $r_{s}<0.25$, considered moderate if $r_{s}=0.25-0.50$, considered good if $r_{\mathrm{s}}=0.50-0.75$ and considered excellent if $r_{\mathrm{s}}>0.75$ [39].

\section{CID (Clinically Important Difference)}

The CID for the PSSD and Vibrometer was determined using receiver operating characteristic (ROC) curves to determine the cut-off point to dichotomize and categorize the sample into 2 groups (responders and non-responders). The SSS was used to determine a cut-off score to discriminate clinically important change based on a cut-off point of 0.5 . The cut off score of 0.5 was based on previous analysis using an ROC curve to determine the minimally clinically important difference for proceed to surgery based on the SSS. The patients were categorized either as responders if change score on the SSS from initial and final follow up was greater than 0.5 (those who responded to orthotic intervention and did not proceed to surgery) or nonresponders if change score was less than 0.5 (those who did not respond to orthotic intervention and proceeded to surgery). The initial scores were taken at baseline. The final follow-up visit was based on scores at 12 weeks. For patients who did not return for their final follow-up, the score at 6 weeks was carried forward as it represented the last known status; and our previous studies have shown that patients who are going to respond to treatment will do so within the first six weeks [40].

ROC curves plotted sensitivity (y-axis) versus 1 -specificity (x-axis). The discriminative ability of the ROC curve is considered good when the area indicated by under the curve exceeds 0.75 [41].

\section{Calculating ES and SRM for Responders to Change}

The ES was calculated by dividing the mean change by the standard deviation of baseline scores [42]. The SRM was calculated by dividing the mean difference of the change scores by the standard deviation of change [43]. The SRM and ES were calculated for both the responder group and the non-responder groups for the PSSD, and the Vibrometer. The change scores were calculated in the same way clinical changes when determining the CID for the PSSD, and Vibrometer. Responsiveness was defined as low if SRM and ES were $<0.5$, moderate if SRM and ES were between 0.5 to 0.8 and large responsiveness if $\geq 0.8[44,45]$.

\section{RESULTS}

Patients were between the ages of 29 to 74 years (mean age of $49 \pm 9$ years). The duration of the symptoms ranged from 1 month -30 years (mean $4 \pm 6$ years) (See Table 1). One person had intermittent symptoms for 30 years, with periods with no symptoms. From 63 patients who completed the SSS and were eligible for this study, 38 patients completed the PSSD, and 22 completed testing with the Vibrometer for both baseline and final follow up. Within this group of patients, the PSSD and the Vibrometer had 1, and 5 patients respectively who had their scores at 6 weeks carried forward to 12 weeks because the 6 week score was the last follow-up.

Table 1. Demographic information of patients.

\begin{tabular}{|l|l|}
\hline Total Number of Participants (n) & 63 \\
\hline Number of Males (n) & 17 \\
\hline Number of Females (n) & 46 \\
\hline Mean Age and Range & $49 \pm 9$ years $(29-70$ years) \\
\hline Symptoms Duration & $4 \pm 6$ years $(1$ month -30 years) \\
\hline Left Hand Affected & 10 \\
\hline Right Hand Affected & 20 \\
\hline Both Hand Affected & 33 \\
\hline Heart Problems & 6 \\
\hline Diabetes & 4 \\
\hline Arthritis & 22 \\
\hline WSIB Compensation Cases & $14(2$ pending $)$ \\
\hline WSIB = Workplace Safety Insurance Board.
\end{tabular}

Table 1a. CTS severity based on electromyography data for median motor distal latency (MDL) and median segmental sensory latency (SDL).

\begin{tabular}{|l|c|c|c|c|}
\hline $\begin{array}{c}\text { Median Motor Distal } \\
\text { Latency (MDL) }\end{array}$ & N & Mean (SD) & Minimum & Maximum \\
\hline \hline Mild (3.8-4.5ms) & 16 & $4.14(0.24)$ & 3.80 & 4.50 \\
\hline Moderate (4.6-6.0ms) & 23 & $5.26(0.40)$ & 4.60 & 6.00 \\
\hline Severe (>6.0ms) & 6 & $8.03(2.25)$ & 6.10 & 11.70 \\
\hline
\end{tabular}

\begin{tabular}{|l|c|c|c|c|}
\hline $\begin{array}{c}\text { Median Segmental } \\
\text { Sensory Latency (SDL) }\end{array}$ & $\mathbf{N}$ & Mean (SD) & Minimum & Maximum \\
\hline \hline Mild (3.0-3.5ms) & 4 & $3.26(0.25)$ & 3.00 & 3.50 \\
\hline Moderate (3.6-4.5ms) & 2 & $4.05(0.35)$ & 3.80 & 4.30 \\
\hline Severe (>4.5ms) & $\mathrm{n} / \mathrm{a}$ & $\mathrm{n} / \mathrm{a}$ & $\mathrm{n} / \mathrm{a}$ & $\mathrm{n} / \mathrm{a}$ \\
\hline
\end{tabular}

$\mathrm{SD}=$ Standard Deviation.

$\mathrm{n} / \mathrm{a}=$ data not available (no severe cases were found with SDL).

The Vibrometer demonstrated moderate correlations to dexterity at most follow-up assessments; and was more strongly related to these tools than was the PSSD (Table 2). The Vibrometer had moderate correlations with Dexterity $(\mathrm{r}=0.36-0.41)$ at all follow up points (Table 2). The PSSD demonstrated low correlation with Dexterity $(\mathrm{r} \leq 0.20)$ at baseline and 12 weeks, but moderate correlation at week 6 $(\mathrm{r}=0.32)$ (Table 2). Neither sensory measure correlated significantly to the DASH $(r=0.09-0.30)$ (Table 2).

The CID for the PSSD was equal $0.15 \mathrm{~g} / \mathrm{mm}^{2}(60 \%$ sensitivity; and 39\% specificity) with an area under the curve of $0.46(0.27,0.64)$ (Fig. 2 and Table 3 ). The ROC curve for the Vibrometer could not be graphed because all 22 individuals who completed vibrometry improved at least 0.5 points on the SSS and the cutoff point did not provide any values for the 
$\mathrm{x}$-axis for 1-specificity (Table 4). The PSSD demonstrated low responsiveness for both responder $(\mathrm{SRM}=0.09$ and $\mathrm{ES}=0.08)$ and non-responder $(\mathrm{SRM}=0.04$ and $\mathrm{ES}=0.06)($ Table 4). The Vibrometer demonstrated moderate responsiveness for responders to treatment $(\mathrm{SRM}=0.61$ and $\mathrm{ES}=0.46)($ Table 4).

Table 2. Spearman correlation between sensory tests and their functional measures.

\begin{tabular}{|c|c|c|c|}
\hline Tools & Time & PSSD $\mathbf{r}_{\mathbf{s}}(\mathbf{C I})$ & Vibrometer $\mathbf{r}_{\mathbf{s}}(\mathbf{C I})$ \\
\hline \hline \multirow{3}{*}{ DASH } & Baseline & $0.09(-0.23-0.43)$ & $0.13(-0.12-0.38)$ \\
\cline { 2 - 4 } & 6 weeks & $0.30(0.01-0.61)$ & $0.21(-0.06-0.48)$ \\
\cline { 2 - 4 } & 12 weeks & $0.10(-0.16-0.37)$ & $0.22(-0.05-0.49)$ \\
\hline \multirow{3}{*}{ Dexterity } & Baseline & $0.20(-0.11-0.51)$ & $0.36^{*}(0.09-0.61)$ \\
\cline { 2 - 4 } & 6 weeks & $0.32 *(0.02-0.62)$ & $0.41^{*}(0.14-0.68)$ \\
\cline { 2 - 4 } & 12 weeks & $-0.02(-0.29-0.25)$ & $0.39^{*}(0.12-0.66)$ \\
\hline
\end{tabular}

PSSD $=$ pressure specified sensory device. DASH $=$ disability of the arm, shoulder, and hand.

* Correlation is significant at $\mathrm{p}<0.05$.

Confidence intervals were calculated with http: //vassarstats.net/rho.html
Table 3. Information on the ROC curves produced.

\begin{tabular}{|c|c|c|}
\hline Properties & $\begin{array}{l}\Delta \text { PSSD } \\
\mathbf{n}=\mathbf{3 8}\end{array}$ & $\begin{array}{c}\Delta \text { Vibrometer } \\
\mathbf{n}=\mathbf{2 2}\end{array}$ \\
\hline External Criterion & \multicolumn{2}{|c|}{$\begin{array}{c}\text { Failed to sufficiently resolve } \\
\text { symptoms - based on } \Delta \mathrm{SSS}=0.5\end{array}$} \\
\hline Cut point for CID & 0.15 & Undefined \\
\hline Area under the curve & $0.46(0.27-0.64)$ & $\mathrm{n} / \mathrm{a}$ \\
\hline Sensitivity & 0.60 & $\mathrm{n} / \mathrm{a}$ \\
\hline Specificity & 0.39 & $\mathrm{n} / \mathrm{a}$ \\
\hline
\end{tabular}

\section{DISCUSSION}

The current study demonstrated that Vibrometry is preferable to PSSD in measuring sensory responses to conservative management of CTS. The Vibrometer was more responsive based on both anchor and distribution based estimates of responsiveness. The correlations between sensory function and hand function were stronger for the

Table 4. Responsiveness for the PSSD, and vibrometer based on CID of $\Delta S S S=0.5$.

\begin{tabular}{|l|c|c|c|c|c|c|}
\hline \multicolumn{1}{|c|}{ Groups } & N & $\begin{array}{c}\text { Mean Baseline Score } \\
\text { (SD) }\end{array}$ & $\begin{array}{c}\text { Mean Post Treatment Score } \\
\text { (SD) }\end{array}$ & $\begin{array}{c}\text { Mean Change } \\
\text { (SD) }\end{array}$ & $\begin{array}{c}\text { SRM } \\
\text { (CI) }\end{array}$ & $\begin{array}{c}\text { ES } \\
\text { (CI) }\end{array}$ \\
\hline \hline PSSD Responder & 15 & $4.13(1.99)$ & $3.83(3.53)$ & $0.30(3.26)$ & $0.09(-0.42-0.60)$ & $0.08(-0.43-0.59)$ \\
\hline PSSD Non Responder & 23 & $7.82(9.47)$ & $7.30(15.29)$ & $0.53(13.48)$ & $0.04(-0.37-0.45)$ & $0.06(-0.35-0.47)$ \\
\hline Vibrometer Responder & 22 & $25.55(19.51)$ & $16.64(14.46)$ & $8.91(14.70)$ & $0.61(0.20-1.02)$ & $0.46(0.05-0.47)$ \\
\hline
\end{tabular}

$\mathrm{SD}=$ standard deviation. $\mathrm{SRM}=$ standardized response mean. $\mathrm{ES}=$ effect size. PSSD $=$ pressure specified sensory device. DASH $=$ disability of the arm, shoulder, and hand. $\mathrm{CI}=$ confidence interval. The overall effect size of all patients was calculated to indicate the overall response to orthotic intervention; SRM and ES were calculated separately for responders and non-responders to indicate responsiveness.

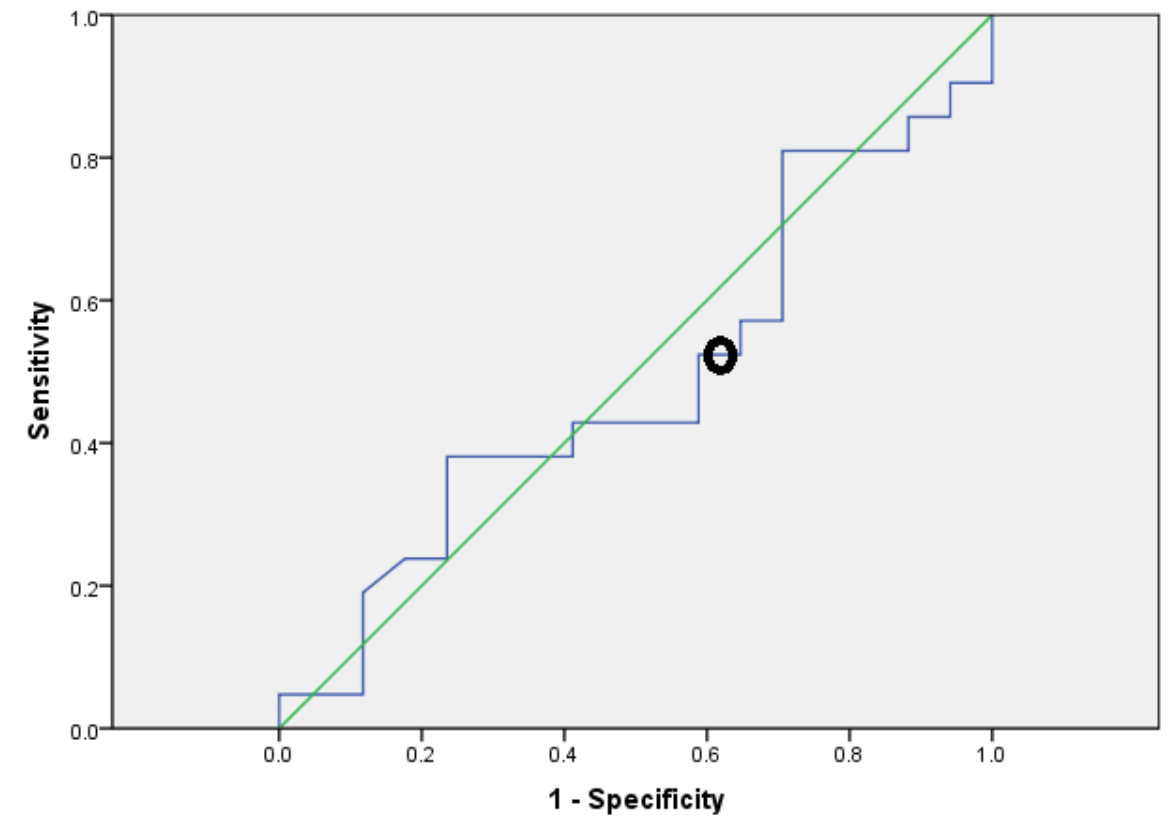

Diagonal segments are produced by ties.

Fig. (2). ROC Curve for determining the probability of a successful outcome based on $\triangle \mathrm{PSSD}$ and CID of 0.5 for the $\Delta$ SSS. Circle represents CID. 
Vibrometer in comparison to the PSSD. Despite some positive findings, neither the PSSD nor Vibrometer discriminated to the extent thought useful for clinical practice. In fact the PSSD was no better than chance at discriminating patients who had symptoms that improved a clinically important amount as (Table 3). The Vibrometer did not provide a defined cut score, as all patients had improved at least 0.5 on the SSS. A larger sample size of patients should be recruited for future studies to increase the number of failures from orthotic intervention to allow for a cut score to be determined for the Vibrometer. Clinicians should be aware that although the Vibrometer may not provide useful information about sensory function, the Vibrometer nor PSSD are sufficient for clinical decisions about whether their patients have made clinically important improvements. Previous literature has stated that vibrometry is insufficient for screening purposes [46]. Since that the Vibrometer shows more promise, further research should consist of a detailed analysis about the use of Vibrometer scores in clinical decision-making. Our study found a significant relationship for sensory scores to dexterity but not to the DASH. Dexterity may be dependent on tactile discrimination as sensory function. The DASH has questions about some aspects of symptoms (numbness/tingling) or function (dressing) that were also assumed to be related to sensory function. The lack of significance correlations may reflect that sensory function is less directly related to DASH items. The Vibrometer demonstrated moderate correlations to dexterity at all follow up points (Table 2). This may have resulted because Meissener corpuscles are affected earlier and recover earlier than other sensory receptors from nerve compression [47]. Any changes in vibration threshold should correlate with changes in dexterity. In addition, there was a moderate correlation between PSSD and Dexterity at 6 weeks, but only low correlation at baseline and 12 weeks. The low correlations between touch threshold and dexterity may have resulted because alterations to sensation are more common than alterations in motor function in mild to moderate CTS cases. Alternations in motor function occur in late stage of chronic compression neuropathies, which are considered severe [46, 48]. Severe cases involving altered motor function would proceed to surgery rather than be treated with conservative management. The finding from this study contrasts results by Melchoir et al. 2006, which found that palmar light touch threshold was correlated to dexterity tasks in patients with peripheral neuropathy [49]. Differences may result because the patients had nerve pathologies from leprosy and not CTS.

Our study results had some limitations. A possible limitation was how we defined clinically important improvement-progression to surgery due to unresolved symptoms. Surgery was used to define whether a patient had clinically important improvement or not from orthotic intervention. We had followed patients for 1 year to determine if patients had surgery for unsuccessful treatment with orthotic intervention. Based on proceeding to surgery, the SSS scores were used to distinguish responders and non-responders from because the SSS is a disease specific tool and would allow for a cutoff point to be set (not just using nominal criteria of proceeding to surgery). Some patients may not have achieved symptom resolution, but may have been reluctant to have surgery. Any misclassification in the external criterion will contribute to a lower CID. However, our responsiveness indices for the SSS suggest this had a minor impact as we differentiated a group with high responsiveness from a group with minimal response on this tool. Since carpal tunnel release is now an out-patient procedure and performed in the outpatient clinic in tertiary care centers, we find that few patients are reluctant to have surgery. However, we cannot eliminate this as a potential factor. It is also possible that some patients went to other centers for surgery, but this is unlikely since our center has a very wide catchment area.

Finally, missing data was a concern since not all patients returned after 6 week follow-up; a number of patients did not complete the sensory tests and the SSS. We experienced a substantial attrition in this study. We do not know if subjects who were better or unchanged were more likely to drop-out, but all patients who returned at 6 weeks and at 12 changed minimally between these 2 time points. Hence, our carry forward approach was the best alternative to estimate the final results for each outcome measure.

\section{CONCLUSION}

This study suggests that the Vibrometer is a better choice for evaluating sensation to represent overall hand function. However, sensory tools should not be used in isolation to make decisions about clinical improvement in CTS until further studies are done to examine different sensory tools and their discriminative ability in larger samples. Despite the common use of sensory evaluation, there remains a large gap in our knowledge of the clinical measurement properties of different tools and test protocol variations.

\section{LIST OF ABBREVIATIONS}

$$
\begin{array}{ll}
\text { CID } & =\text { Clinically Important Difference } \\
\text { CTS } & =\text { Carpal Tunnel Syndrome } \\
\text { DASH } & =\text { Disability of the Arm, Shoulder, and Hand } \\
\text { EMG } & =\text { Electromyography } \\
\text { ES } & =\text { Effect Size } \\
\text { ICC } & =\text { Intra class Correlation Coefficient } \\
\text { PSSD } & =\text { Pressure Specified Sensory Device } \\
\text { ROC } & =\text { Receiver Operating Characteristic } \\
\text { SRM } & =\text { Standardized Response Mean } \\
\text { SSS } & =\text { Symptom Severity Scale }
\end{array}
$$

\section{CONFLICT OF INTEREST}

The authors confirm that this article content has no conflict of interest.

\section{ACKNOWLEDGEMENTS}

Special thanks Jan Smith who helped with data collection and data entry. 


\section{REFERENCES}

[1] Atroshi I, Gummesson C, Johnsson R, Ornstein E, Ranstam J, Rosén I. Prevalence of carpal tunnel syndrome in a general population. JAMA 1999; 282(2): 153-8.

[2] Luckhaupt SE, Dahlhamer JM, Ward BW, Sweeney MH, Sestito JP, Calvert GM. Prevalence and work-relatedness of carpal tunnel syndrome in the working population, United States, 2010 national health interview survey. Am J Ind Med 2013; 56(6): 615-24.

[3] Tanaka S, Wild DK, Seligman PJ, Halperin WE, Behrens VJ, PutzAnderson V. Prevalence and work-relatedness of self-reported carpal tunnel syndrome among US workers: analysis of the occupational health supplement data of 1988 National Health Interview Survey. Am J Ind Med 1995; 27(4): 451-70.

[4] Zakaria D. Rates of carpal tunnel syndrome, epicondylitis, and rotator cuff claims in Ontario workers during 1997. Chronic Dis Can 2004; 25(2): 32-9.

[5] Zakaria D, Robertson J, MacDermid J, Hartford K, Koval J. Workrelated cumulative trauma disorders of the upper extremity: navigating the epidemiologic literature. Am J Ind Med 2002; 42(3): 258-69.

[6] Feuerstein M, Miller VL, Burrell LM, Berger R. Occupational upper extremity disorders in the federal workforce: prevalence, health care expenditures, and patterns of work disability. J Occup Environ Med 1998; 40(6): 546-55.

[7] Werner RA, Andary M. Carpal tunnel syndrome: pathophysiology and clinical neurophysiology. J Clin Neurophysiol 2002; 113(9): 1373-81.

[8] Kerwin G, Williams CS, Seiler JG $3^{\text {rd }}$. The pathophysiology of carpal tunnel syndrome. Hand Clin 1996; 12(2): 243-51.

[9] Gerritsen AAM, de Vet HCW, Scholten RJPM, Bertelsmann FW, de Krom MC, Bouter LM. Splinting vs surgery in the treatment of carpal tunnel syndrome. JAMA 2002; 288(10): 1245-51.

[10] Gerritsen AA, de Krom MC, Struijs MA, Scholten RJ, de Vet HC, Bouter LM. Conservative treatment options for carpal tunnel syndrome: a systematic review of randomised controlled trials. J Neurol 2002; 249(3): 272-80.

[11] Gerritsen AA, de Vet HC, Scholten RJ, van Tulder MW, Bouter LM. Enabling meta-analysis in systematic reviews on carpal tunnel syndrome. J Hand Surg Am 2002; 27(5): 828-32.

[12] Gerritsen AA, Uitdehaag BM, van Geldere D, Scholten RJ, de Vet HC, Bouter LM. Systematic review of randomized clinical trials of surgical treatment for carpal tunnel syndrome. Br J Surg 2001; 88(10): 1285-95

[13] Gerritsen AAM, De Vet HCW, Scholten RJPM, Bertelsmann FW, De Krom MCTFM, Bouter LM. Greater clinical effects on carpal tunnel syndrome with surgery than with splinting: results of a randomised clinical trial. Ned Tijdschr Geneeskd 2002; 146(45): 2153-6.

[14] Rempel D, Evanoff B, Amadio PC, et al. Consensus criteria for the classification of carpal tunnel syndrome in epidemiologic studies. Am J Public Health 1998; 88(10): 1447-51.

[15] MacDermid J. Accuracy of clinical tests used in the detection of carpal tunnel syndrome: a literature review. J Hand Ther 1991; 4(4): 169-76.

[16] MacDermid JC, Kramer JF, Roth JH. Decision making in detecting abnormal Semmes-Weinstein monofilament thresholds in carpal tunnel syndrome. J Hand Ther 1994; 7(3): 158-62.

[17] MacDermid J, Kramer J, McFarlane R, Roth J. Inter-rater agreement and accuracy of clinical tests used in diagnosis of carpal tunnel syndrome. Work 1997; 8(1): 37-44.

[18] MacDermid JC, Doherty T. Clinical and electrodiagnostic testing of carpal tunnel syndrome: a narrative review. J Orthop Sports Phys Ther 2004; 34(10): 565-88.

[19] MacDermid JC, Wessel J. Clinical diagnosis of carpal tunnel syndrome: a systematic review. J Hand Ther 2004; 17(2): 309-19.

[20] Thonnard JL, Saels P, den Bergh PV, Lejeune T. Effects of chronic median nerve compression at the wrist on sensation and manual skills. Exp Brain Res 1999; 128(1): 61-4.

[21] Bell-Krotoski J, Weinstein S, Weinstein C. Testing sensibility, including touch-pressure, two-point discrimination, point localization, and vibration. J Hand Ther 1993; 6(2): 114-23.
[22] Levine DW, Simmons BP, Koris M, et al. A self-administered questionnaire for the assessment of severity of symptoms and functional status in carpal tunnel syndrome. J Bone Joint Surg Am 1993; 75: 1585-92.

[23] Liang H, Wang Y, Pan S, Wang T, Huang T. Asymptomatic median mononeuropathy among men with chronic paraplegia. Arch Phys Med Rehabil 2007; 88(9): 1193-7.

[24] Yost KJ, Eton DT. Combining distribution- and anchor-based approaches to determine minimally important differences. Eval Health Prof 2005; 28(2): 172-91.

[25] Guyatt GH, Osoba D, Wu AW, Wyrwich KW, Norman GR, Clinical Significance Consensus Meeting Group. Methods to explain the clinical significance of health status measures. Mayo Clin Proc 2002; 77(4): 371-83.

[26] Kamper SJ, Maher CG, Mackay G. Global rating of change scales: a review of strengths and weaknesses and considerations for design. J Man Manip Ther 2009; 17(3): 163-70.

[27] Shi Q, MacDermid JC. Is surgical intervention more effective than non-surgical treatment for carpal tunnel syndrome? a systematic review. J Orthop Surg Res 2011; 6(1): 1-9.

[28] Thonnard JL, Saels P, den Bergh PV, Lejeune T. Effects of chronic median nerve compression at the wrist on sensation and manual skills. Exp Brain Res 1999; 128(1): 61-4.

[29] Caliandro P, La Torre G, Aprile I, et al. Distribution of paresthesias in Carpal Tunnel Syndrome reflects the degree of nerve damage at wrist. Clin Neurophysiol 2006; 117(1): 228-31.

[30] Dellon AL, Keller KM. Computer-assisted quantitative sensorimotor testing in patients with carpal and cubital tunnel syndromes. Ann Plast Surg 1997; 38(5): 493-502.

[31] Hubbard MC, MacDermid JC, Kramer JF, Birmingham TB Quantitative vibration threshold testing in carpal tunnel syndrome: analysis strategies for optimizing reliability. J Hand Ther 2004; 17(1): 24-30

[32] McConnell SG, Beaton DE, Bombardier C. The DASH outcome measure user's manual. $1^{\text {st }}$ ed. Toronto, Ontario: Institute for Work \& Health 1999.

[33] Kotsis SV, Lau FH, Chung KC. Responsiveness of the Michigan hand outcomes questionnaire and physical measurements in outcome studies of distal radius fracture treatment. J Hand Surg Am 2007; 32(1): 84-90.

[34] Beaton DE, Katz JN, Fossel AH, Wright JG, Tarasuk V, Bombardier C. Measuring the whole or the parts? validity, reliability, and responsiveness of the Disabilities of the Arm, Shoulder and Hand outcome measure in different regions of the upper extremity. J Hand Ther 2001; 14(2): 128-46.

[35] Tucker AT, White PD, Kosek E, et al. Comparison of vibration perception thresholds in individuals with diffuse upper limb pain and carpal tunnel syndrome. Pain 2007; 127(3): 263-9.

[36] MacDermid JC, Mulè M. Concurrent validity of the NK hand dexterity test. Physiother Res Int 2001; 6(2): 83-93.

[37] Turgeon TR, MacDermid JC, Roth JH. Reliability of the NK dexterity board. J Hand Ther 1999; 12(1): 7-15.

[38] Finch E, Brooks D, Stratford PW, Mayo NE. Physical rehabilitation outcome measures: a guide to enhanced clinical decision making. $2^{\text {nd }}$ ed. Hamilton: BC Decker Inc 2002.

[39] Portney LG, Watkins MP, Eds. Foundations of clinical research applications to practice. $2^{\text {nd }}$ ed. Upper Saddle River, New Jersey: Prentice Hall Health 2000

[40] Boyd KU, Sgan B, Ross DC, Richards RS, Roth JH, MacDermid J. Outcomes in carpal tunnel syndrome: symptom severity, conservative management and progression to surgery. Clin Invest Med 2005; 28(5): 254-60.

[41] Worster A, Fan J, Upadhye S. Understanding receiver operating characteristic (ROC) curves. CJEM 2006; 8(1): 19-20.

[42] Kazis LE, Anderson JJ, Meenan RF. Effect sizes for interpreting changes in health status. Med Care 1989; 27(Suppl 3): S178-89.

[43] Liang MH, Larson MG, Cullen KE, Schwartz JA. Comparative measurement efficiency and sensitivity of five health status instruments for arthritis research. Arthritis Rheumat 2005; 28(5): 542-7.

[44] Cohen J. Statistical power analysis for the behavioral sciences. $2^{\text {nd }}$ ed. New Jersey, USA: Lawrence Erlbaum 1988. 
[45] Cohen J. Statistical power analysis for the behavioral sciences. $1^{\text {st }}$ ed. New Jersey, USA: Lawrence Erlbaum 1977.

[46] Werner RA, Andary M. Carpal tunnel syndrome: pathophysiology and clinical neurophysiology. Clin Neurophysiol 2002; 113(9): 1373-81.

[47] Krishnamurti A, Kanagasuntheram R, Vij S. Failure of reinnervation of Pacinian corpuscle after nerve crush. Acta Neuropathol 1973; 23(4): 338-41.
[48] Mackinnon SE. Pathophysiology of nerve compression. Hand Clin 2002; 18(2): 231-41.

[49] Melchior H, Vatine JJ, Weiss PL. Is there a relationship between light touch-pressure sensation and functional hand ability? Disabil Rehabil 2007; 29(7): 567-75.

(C) Cheung et al.; Licensee Bentham Open.

This is an open access article licensed under the terms of the Creative Commons Attribution Non-Commercial License (http://creativecommons.org/licenses/by-nc/3.0/) which permits unrestricted, non-commercial use, distribution and reproduction in any medium, provided the work is properly cited. 